\title{
L'HOMME L'Homme
}

Revue française d'anthropologie

160 | octobre-décembre 2001

Droit, coutume, mémoire

\section{Patrick Saurin, Teocuicatl. Chants sacrés des anciens}

Mexicains

Paris, Publications scientifiques du Muséum, 1999, 275 p., annexe, bibl., index, ill., cartes (« Mémoires de l'Institut d'ethnologie » 36)

\section{Serge Gruzinski}

\section{OpenEdition}

12 Journals

Édition électronique

URL : http://journals.openedition.org//homme/7849

DOI : $10.4000 /$ /homme.7849

ISSN : 1953-8103

Éditeur

Éditions de l'EHESS

Édition imprimée

Date de publication : 1 janvier 2001

Pagination : 243-245

ISBN : 2-7132-1391-6

ISSN : 0439-4216

Référence électronique

Serge Gruzinski, «Patrick Saurin, Teocuicatl. Chants sacrés des anciens Mexicains », L'Homme [En ligne], 160 | octobre-décembre 2001, mis en ligne le 31 mai 2007, consulté le 22 septembre 2020. URL :

http://journals.openedition.org//homme/7849; DOI : https://doi.org/10.4000//homme.7849

Ce document a été généré automatiquement le 22 septembre 2020.

(c) École des hautes études en sciences sociales 


\section{Patrick Saurin, Teocuicatl. Chants sacrés des anciens Mexicains}

Paris, Publications scientifiques du Muséum, 1999, 275 p., annexe, bibl., index, ill., cartes (« Mémoires de l'Institut d'ethnologie » 36)

\section{Serge Gruzinski}

PATRICK SAURIN s'est penché sur l'un des nombreux manuscrits laissés par le franciscain Bernardino de Sahagùn: les teocuicatl, ces hymnes sacrés composés en l'honneur des anciens dieux mexicains. Vraisemblablement d'origine mexica, ces chants ont été rassemblés à Tepepulco vers 1560 . S'agit- il de textes archaïques comme la critique s'est accordée à le penser ? L'auteur propose d'y voir, au moins pour partie d'entre eux, des hymnes portant la «marque d'une réécriture, voire d'une écriture récente » (p. 45) et, par conséquent, influencés par les pratiques de la société mexica à la fin $\mathrm{du} \mathrm{XV}^{\mathrm{e}}$ siècle. Il faut avouer que ces témoignages ne sont guère aisés à lire et à interpréter. Les traductions établies par Eduard Seler, Angel María Garibay et Thelma Sullivan sont loin d'avoir fait la lumière sur des textes essentiels pour la compréhension des rites et des cultes mexicas. Nous sommes donc en présence d'un corpus difficile à explorer tant il est loin d'être exhaustif et nous parvient à jamais mutilé, dépourvu qu'il est des musiques, des chants et des danses qui l'accompagnaient. C'est le mérite de Patrick Saurin d'avoir tenté une nouvelle traduction en proposant de nouvelles interprétations, comme celles qui suggèrent que l'hymne de Huitzilopochtli serait le Tlaxotecayotl chanté lors de la fête Panquetzalilztli, alors que le Chant de Xipe Totec correspondrait à une « réécriture aztèque » d'un rite huastèque. L'auteur n'hésite pas à reconnaître les limites auxquelles se heurte souvent son analyse, quand, par exemple, il s'interroge sur l'identification des amanteca et des pipiteca qui interviennent à la fin du Chant de Huitzilopochtli. Sa recherche mobilise une multitude d'informations tirées des sources les plus diverses, qu'elles soient écrites ou peintes, d'origine préhispanique ou coloniale, pour éclairer tel ou tel aspect des chants. L'archéologie et l'ethnographie sont largement mises à contribution pour corroborer des interprétations ou éclaircir des obscurités. Au fil du livre, Patrick Saurin parcourt une bonne partie des mythologies amérindiennes, nahua et même maya, en établissant des rapprochements suggestifs comme celui qu'il fait entre une planche du Codex Borgia et le Chant de 
Xochipilli. Signalons également l'intérêt de la confrontation introduite entre ce dernier hymne et celui de Nezahualcoyotl, car elle contribue à dégager des ordres d'expression distincts, des dissonances au sein d'une pensée dont l'homogénéité est souvent exagérée.

2 La lecture de l'ouvrage laisse pourtant une certaine frustration: on aurait souhaité que ce livre renouvelle davantage notre connaissance des croyances mexicas au lieu de se borner souvent à des confirmations ou à des rappels concernant la prééminence accordée aux divinités guerrières, la valorisation de la guerre, ou encore tel ou tel schéma cosmogonique. Si les remarques concernant la fusion de trois plans distincts divin, mythique et terrestre - dans le Chant de Tlaloc sont bien venues, des questions de fond restent posées. Ces textes recueillis une quarantaine d'années après la conquête espagnole, auprès d'informateurs indigènes forcément christianisés, n'auraient-ils pas subi d'autres "réécritures »? La chute de la domination mexica a changé les rapports de forces dans la vallée de Mexico et le regard que les Indiens jetaient sur le passé préhispanique s'en est souvent trouvé modifié.

Quant à la méthode consistant à puiser sans cesse dans des textes d'origine fort variée, rarement contextualisés et insuffisamment critiqués, elle n'est pas sans soulever quelques difficultés. Il aurait été intéressant de partir du rapprochement établi entre des invocations recueillies au XVII ${ }^{\mathrm{e}}$ siècle, en pleine époque coloniale, et le Chant d'Amimitl, rédigé dans un nahuatl archaïque: quelles conclusions tirer de cette apparente continuité qui traverse la conquête espagnole ? S'il est vrai que ce mode d'interprétation qui procède par juxtaposition est souvent la règle et la panacée dans les études de textes de l'Ancien Mexique, on peut se demander s'il ne mériterait pas d'être remis en cause. Si passer du monde olmèque au monde maya pour interpréter un texte nahuatl fixé au $\mathrm{XvI}^{\mathrm{e}}$ siècle témoigne d'un esprit curieux, ces acrobaties ne manqueront pas d'étonner l'historien préoccupé de distinguer les époques, les sociétés et les cultures, et pas forcément acquis d'avance à l'idée de l'existence d'une « culture mésoaméricaine » (p. 127).

4 On aurait également souhaité une approche plus dynamique de la pensée nahua, qui dépasse les limites d'une exégèse pour dégager des apports véritablement novateurs. Un examen plus approfondi de la manière dont les chants sacrés absorbent des faits historiques - la piste nous est pourtant suggérée page 199, à propos du Chant d'Otontecuhtli- ne nous aurait-il pas livré des indications précieuses sur le fonctionnement de la mémoire nahua et ses rapports au passé ?

5 Les conclusions consacrées à l'analyse de la parole sacrée des anciens Mexicains s'attachent à dégager des traits communs au sein du corpus des teocuicatl. Elles incitent l'auteur à le subdiviser en trois catégories : la chasse, la guerre et le cycle du maïs et de la vie. Comme dans le reste de l'ouvrage, Saurin reste sensible à la complexité des matières qu'il explore et se garde d'introduire des catégories trop tranchées. Mais cette prudence et cette honnêteté de la démarche l'empêchent sans doute de tirer de ces extraordinaires documents des aperçus plus neufs et plus suggestifs, comme ceux qui l'entraînent un bref moment vers les Mythologiques de Claude Lévi-Strauss et la Phénoménologie de l'esprit de Hegel. Signalons au lecteur que, outre quelques belles planches, il trouvera en annexe un dossier fort utile qui éclaire le choix des traductions fait par l'auteur. Cet appendice inclut également la traduction des gloses du commentateur mexica. 


\section{AUTEUR}

SERGE GRUZINSKI

CNRS-EHESS, Centre de recherche sur les mondes américains, Paris. 